\title{
BIBLIOGRAPHIE
}

LAHACHe et MARRe. Beurre de vache et graisse de coco. Editeur : Maloine, Paris.

E. Grognard. $2^{\mathrm{e}}$ Congrès d'Hygiène alimentaire, Bruxelles $1910,2^{\mathrm{e}}$ volume, p. 227.

I. Wauters. $2^{\text {e }}$ Congrès d'Hygiène alimentaire, Bruxelles, 1910. $2^{e}$ volume, p. 181.

Van Engelen et P. Wauters. Enquête sur les beurres de Bouchout. Bull. de l'Agriculture belge, 1899.

Dr Swaving. Le Lait, XIII, mars 1933.

$-2^{\mathrm{e}}$ Congrès de Laiterie, Bruxelles, $1^{\text {re }}$ Section.

LAXA. Annales des Falsifications, 1932, p. 529.

Prof. Polonowski et Dr Thomas. Le Lait, XIII, mars, avril, mai 1933, pp. $249,466,583$.

Dr Balavoine. Le Lait, XIX, décembre 1939, p. 1027.

Dr Van Beneden et Prof. Vivario. Le Lait, XVI, février 1936, p. 113.

Hотом. Beurres anormaux. Journal de Pharmacie d'Anvers, 1904.

- Beurres purs, beurres falsifiés. Journal de Pharmacie d'Anvers, 1906.

- Les indices de réfraction. Annales des Falsifications, janvier 1909.

- Beurres du pays de Herve (1re ótude). Journal de Pharmacie de Belgique ; $2{ }^{\circ}$ étude. Journal de Pharmacie de Belgique, 1930, nos 22-24 et 36; 25-24.

- Glycérides de beurre. Journal de Pharmacie de Belgique, 1932, no 33.

- Beurres anormaux. Annales des Falsifications, août 1913.

\section{L'UTILISATION DU SÉRUM}

\author{
par \\ G. GENIN \\ Ingénieur E. P. C.
}

On sait que l'utilisation industrielle des sous-produits de l'industrie laitière est liée à un certain nombre de facteurs économiques qui ont trait à la nature des produits que l'on peut isoler, aux dépenses qu'entraîne la séparation de ces produits et aux cours auxquels on peut les revendre. Lorsqu'on utilise dans une laiterie du lait entier en vue de le transformer en produits marchands, on admet qu'on peut en tirer environ $10 \%$ de crème, $2,8 \%$ de caséine et $75 \%$ de sérum. Ce sérum est essentiellement constitué d'eau, de lactose, de lactalbumine et de vitamine G. La méthode la plus simple pour la récupération de ce sérum est de le concentrer de façon à obtenir un liquide épais que l'on mélange avec de la poudre de sérum desséché, de façon à obtenir une substance de consistance granulaire qui est à son tour desséchée et pulvérisée. Cette poudre, qui est la poudre de sérum du commerce, est essentiellement utilisée pour la nourriture des animaux et de la volaille. Malheureusement, le prix de vente de cette poudre est très faible et il est rare que la récupération de 
ce produit couvre les dépenses qu'entraîne cette opération. Il faut pour que l'opération soit payante que l'utilisation du sérum sous cette forme évite les traitements des eaux résiduaires, traitements toujours coûteux, dont la suppression vient donc en déduction du prix de revient de la poudre de sérum.

Toutefois, depuis quelques années, l'état de la question s'est modifié. En effet, on a découvert qu'il existait dans le sérum du lait des produits très importants, comme le lactose, susceptibles de servir de point de départ à une industrie chimique nouvelle. Nous avons donné à plusieurs reprises des extraits et même des reproductions intégrales des travaux effectués aux Etats-Unis sur la récupération des sous-produits du sérum et nous rappellerons que le lactose peut être transformé en acide lactique et que les esters de cet acide sont des produits précieux pour la fabrication de nouvelles résines synthétiques.

C'est ce nouveau point de vue dans l'utilisation du sérum qui a incité certaines usines américaines à réaliser industriellement l'extraction des sous-produits du sérum et nous allons décrire dans ce qui suit l'installation créée récemment par une firme de l'Etat de New-York, la "Sheffield By-Products Company", en empruntant cette description à une étude publiée par W. E. STRINGER dans la revue "Food Industries". Ultérieurement, nous indiquerons comment le lactose brut peut être à son tour purifié.

-On admet qu'en moyenne la.composition du lait est donnée par les chiffres suivants :

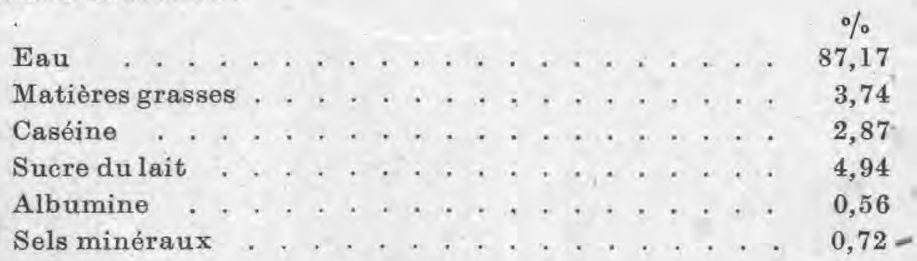

Après passage du lait aux écrémeuses, on obtient du lait écrémé, pratiquement exempt de matières grasses, qui constitue le point de départ de l'extraction des sous-produits. Dans les usines Sheffield, la caséine est séparée par un procédé particulier à cette usine dans lequel la précipitation se fait par l'acide chlorhydrique. On obtient done du sérum contenant en solution du lactose, de l'albumine, des sels minéraux et des vitamines.

Le sérum provenant de l'extraction de la caséine est alors envoyé dans des baes en fer dénommés réservoirs de coagulation. L'acidité du sérum lorsqu'il pénètre dans ces baes est d'environ $0,40 \%$ calculé en acide lactique. Par addition d'un lait de chaux, on abaisse cette acidité à environ $0,05 \%$ en acide lactique. A ce 


\section{SCHEMA DUUNE INSTALLATION OE FABRICATION DE LACTOSE BRUT ET D'ALIMENT POUR VOLAILLES}
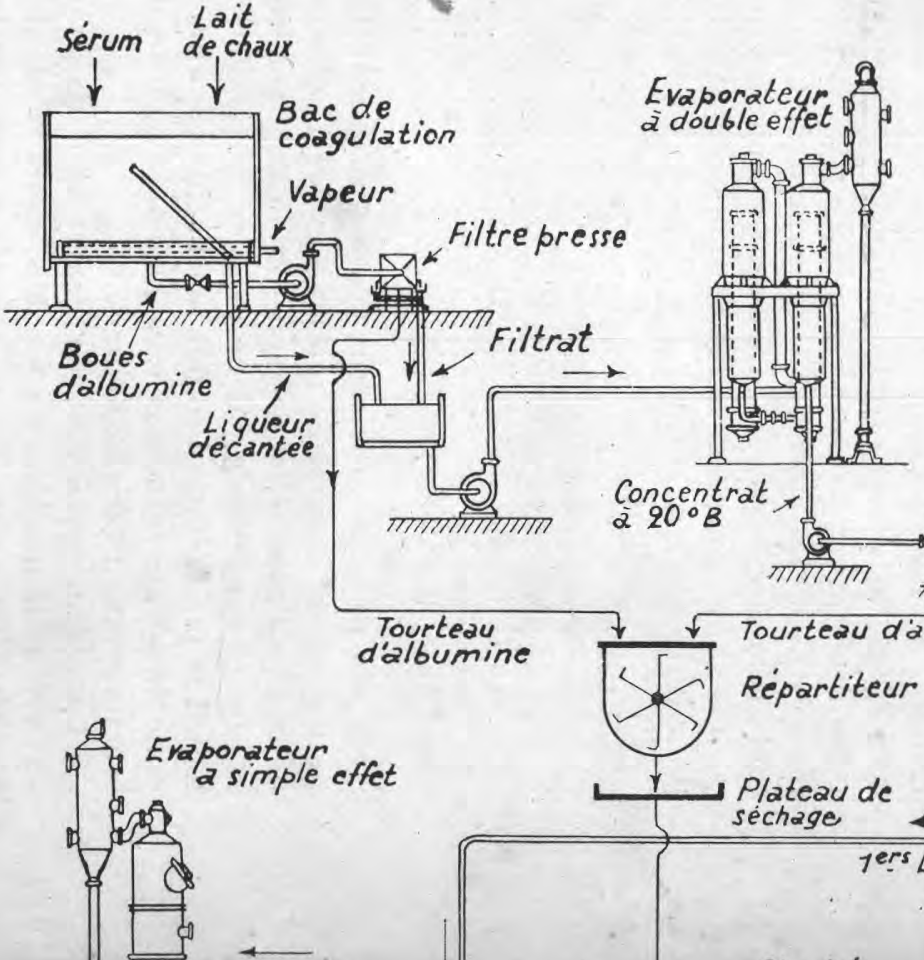


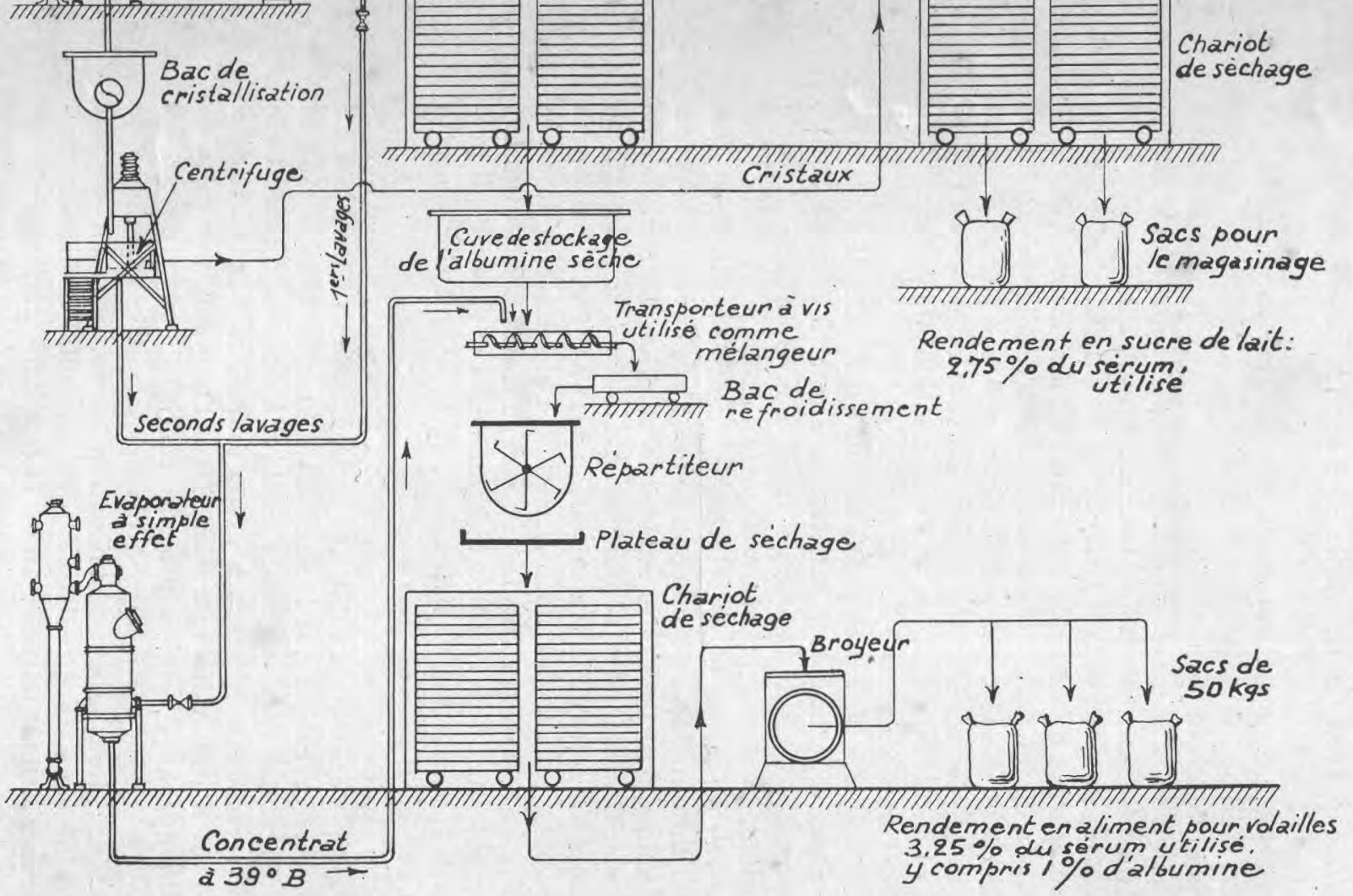


moment, on introduit dans le sérum par l'intermédiaire d'un tuyau perforé situé au fond du bac de la vapeur vive, afin de porter le liquide à l'ébullition.

Sous l'influence de ce traitement combiné par la chaux et par la chaleur, l'albumine se trouve coagulée et transformée en une forme insoluble, ce qui permet sa séparation. En quelques minutes en effet l'albumine se rassemble sous forme de boue que l'on recueille au fond du réservoir. Il suffit done de décanter le liquide clair surnageant jusqu'à ce que l'on arrive au niveau de la couche formée par les boues de l'huile. La liqueur décantée est à ce moment envoyée dans un appareil d'évaporation à double effet, tandis que les boues d'albumine sont dirigées par l'intermédiaire d'une pompe vers un filtre-presse afin de réaliser une séparation plus complète de l'albumine, que l'on séchera ensuite, et de la solution qui est ajoutée à celle passant dans l'appareil d'évaporation.

A l'usine Sheffield, cet appareil est du type vertical à concentration rapide; il permet de concentrer la liqueur à environ $20^{\circ} \mathrm{Bé}$, ce qui correspond à une teneur en sucre d'environ $30 \%$. A cette concentration, la teneur en sucre est insuffisante pour qu'il y ait début de cristallisation et la solution concentrée peut done être dirigée par l'intermédiaire d'une pompe dans une euve de stockage.

A ce moment, il faut faire subir à la solution concentrée une nouvelle clarification pour séparer les matières insolubles qui se sont formées par suite de la concentration de la liqueur. Ces matières insolubles sont çonstituées essentiellement d'albumine et de sucre de lait et la clarification s'effectue au moyen d'un filtre-presse. Les tourteaux provenant de ce filtre sont mélangés avec ceux qui proviennent de la séparation de l'albumine, après coagulation de cette dernière, comme il a été dit plus haut.

Le sirop clarifié est alors dirigé vers un nouvel appareil d'évaporation à simple effet où il subit une concentration finale. C'est au cours de cette opération que le sirop est concentré dans le vide et c'est à l'opérateur, qu'une grande pratique a formé, qu'incombe le soin de déterminer le moment à partir duquel la cristallisation a commencé et d'arrêter l'évaporation. Dans l'appareil, par suite de l'ébullition du sirop, celui-ci projette continuellement de petites quantités de liquide sur le regard placé à la partie supérieure de l'évaporateur; lorsque la cristallisation commence, un ouvrier bien entraîné constate que l'aspect des particules de liquide projetées sur le regard est modifié. Au fur et à mesure que l'on prolonge l'opération, les cristanx qui se forment sur le regard en verre grossissent et lorsque les cristaux ont atteint la grosseur voulue, on fait tomber tout le contenu de l'évaporateur dans un mélangeur.

Ce mélangeur est situé juste au-dessous de l'orifice de décharge 
de l'évaporateur et il est constitué par un réservoir rectangulaire portant un fond arrondi. Un agitateur à arbre horizontal communique une certaine agitation au sirop, agitation très complète car l'appareil porte en réalité des hélices tournant en sens inverse et à la vitesse d'environ 6 à 10 tours par minute. Le mouvement ainsi créé dans l'appareil est suffisant pour agiter continuellement les eaux-mères d'une part et les cristaux, ce qui empêche la prise du mélange avant que l'opération de lavage soit complètement terminée.

De ce mélangeur, les cristaux de sucre et les eaux-mères s'écoulent à l'intérieur d'un tube de cuivre et par simple gravité dans un appareil centrifuge à baquet où s'effectue le lavage proprement dit. Cet appareil, du type Hepworth, est, constitué essentiellement d'un baquet cylindrique perforé tournant à la vitesse d'environ 600 tours par minute. C'est ici que s'effectue la séparation des cristaux de sucre, du sirop, sous l'influence de la force centrifuge. En faisant pénétrer dans l'appareil un petit courant d'eau, et sans arrêter le mouvement de rotation, on procède à un lavage des cristaux et à l'élimination des impuretés solubles. Lorsque ce lavage est terminé, on porte la vitesse du baquet à 1.200 tours par minute, de façon à éliminer rapidement une nouvelle quantité d'eau. Finalement, le gâteau de sucre qui s'est rassemblé sur les parois du baquet est détaché au moyen d'un couteau, en faisant tourner lentement le baquet.

Les cristaux de sucre brut provenant de l'appareil centrifuge sont alors rapidement répartis à la main sur des plateaux de séchage ; ces plateaux sont placés sur des chariots transporteurs que l'on fait circuler dans des tunnels sécheurs.

Les eaux-mères séparées de l'appareil centrifuge, ainsi que les eaux de lavage sont réunies pour être à nouveau concentrées et les cristaux bruts que l'on obtient au cours de cette opération sont généralement destinés à la nourriture de la volaille.

L'expérience a en effet montré que les frais de récupération et de purification du lactose, lorsque l'on veut que cette opération soit complète, sont très élevés. Il est préférable de ne pas chercher à récupérer les dernières parties de sucre et à ajouter ce produit aux résidus destinés à la fabrication des produits alimentaires pour la volaille.

Cette façon de faire semble apparemment entraîner une diminution du rendement en sucre de lait, mais en réalité cette perte est beaucoup moins importante qu'on pourrait le penser puisque ce sucre se retrouve dans les aliments pour volailles, qui doivent obligatoirement contenir une proportion garantie de lactose. L'intérêt de ce procédé ressort d'ailleurs de la comparaison de 
quelques chiffres : en effet, dans l'ancien procédé, alors qu'on cherchait à obtenir un rendement maximum en lactose, le rendement en matières solides totales ne dépassait pas $4 \%$, alors que, par la méthode utilisée à Sheffield, le rendement en matières solides totales atteint $6 \%$, avec cependant des frais de production moindres.

On peut expliquer le rendement relativement faible en sucre /de lait qu'on obtient par le fait que les sels contenus dans lo lait ainsi que les protéines solubles présentent l'inconvénient d'empêcher la cristallisation du lactose. Si, par conséquent, les eaux de lavage provenant du traitement du lactose brut retournaient continuellement dans les appareils d'évaporation, les liqueurs s'enrichiraient en sels et en protéines et la séparation du sucre de lait deviendrait impossible. II y a donc avantage à diriger ces eaux de lavage vers les appareils utilisés pour la fabrication des aliments pour volailles, d'autant plus que ces eaux contiennent des produits dont la valeur nutritive a été nettement reconnue. L'incorporation de ces produits dans les aliments pour volailles permet donc d'obtenir des produits de meilleure qualité, d'assurer une récupération totale des matières solides du lait, avec des frais les plus réduits possible.

La pratique courante est donc de ne faire cristalliser qu'une seule fois les eaux de lavage provenant de l'appareil centrifuge, en vue de séparer le sucre de lait qu'elles peuvent contenir. Le résidu sera ensuite dirigé vers l'atelier de fabrication des produits alimentaires pour volailles. Pour effectuer cette opération, ces eaux de lavage sont donc dirigées dans un appareil évaporateur fonetionnant dans le vide ; lorsque la concentration désirée est atteinte, on verse les eaux concentrées dans des bacs de refroidissement où la cristallisation du lactose s'effectue. Les cristaux et les eaux-mères sont séparés par centrifugation, on obtient ainsi après lavage des cristaux bruts de lactose et des eaux de lavage.

Ces dernières contiennent des quantités substantielles de sucre de lait, de sels du lait, de protéines solubles et de vitamines. L'expérience de la pratique a montré que l'utilisation la plus économique de ces sous-produits était la fabrication d'aliments pour volailles. La fabrication comporte tout d'abord une concentration des eaux de lavage dans le vide, de façon á obtenir une liqueur concentrée marquant environ $39^{\circ}$ Bé. Cette liqueur concentrée s'écoule de l'appareil évaporateur dans un mélangeur où on lui ajoute l'albumine sèche dont la préparation a été décrite au début de cette note. Les deux produits : albumine sèche et eaux de lavage concentrées, sont intimement mélangés dans un appareil constitué par un cylindre horizontal portant à l'intérieur une vis transporteuse jouant le rôle de mélangeur. L'alimentation en albumine sèche se fait également par vis transporteuse et on parvient ainsi à réaliser un mélange 
très intime du sucre et de l'albumine, la composition de ce mélange étant réglée par la vitesse d'introduction des eaux concentrées.

L'albumine sèche absorbe une quantité considérable d'humidité de la liqueur sucrée, de telle sorte que le mélange final présente une consistance granulaire. On laisse ce mélange "durcir", ce qui se fait par cristallisation du sucre, et on le répand uniformément sur des plateaux de séchage au moyen d'un répartiteur.

Le dispositif de répartition est constitué par un tambour en fer muni d'un fond perforé. Au moyen d'un agitateur rotatif situé à l'intérieur du tonneau, on oblige la poudre de sérum à traverser les perforations du tambour et à tomber sur un plateau de séchage. Les plateaux ainsi garnis sont empilés sur.les chariots et placés dans des tunnels sécheurs où la température est réglée entre 60 et $70^{\circ}$, le séchage demandant 3 à 4 heures. Grâce à la texture granulaire du mélange d'albumine et de sucre, il est possible de répartir uniformément sur les plateaux la matière à sécher, ce qui permet ultérieurement de réaliser un séchage uniforme.

A la sortie des sécheurs, le contenu des plateaux est versé dans une trémie qui alimente un broyeur à marteaux où le produit sec est pulvérisé. Le produit en poudre est alors ensaché dans des saes doublés intérieurement de papier imperméabilisé, chaque sac contenant 50 kilogrammes de matière.

Ce produit fini a un aspect plaisant et est coloré en jaune clair, sa teneur garantie en lactose est de $30 \%$ et elle est de $25 \%$ en protéines.

En ce qui concerne la question des rendements, on notera que le rendement en matière sèche totale est d'environ $6 \%$, ce rendement se répartissant en lactose brut, en albumine et en aliments pour volailles. Il est de $2,75 \%$ en lactose brut et de $1 \%$ environ en albumine, ce dernier produit étant ultérieurement utilisé également pour la nourriture des volailles.

\title{
LE LAIT FRAIS PASTEURISÉ IRRADIÉ A L'ABRI DE L'AIR ET LA VITAMINE D INDICATIONS MÉdICALES - POSOLOGIE
}

\author{
par \\ Docteur JEAN VIEILLY \\ Vétérinaire.
}

A l'Exposition de New-York (1939) on a présenté, dans la ferme électrifiée, une étable modèle où le lait, avant d'être mis en bouteilles stérilisées, est pasteurisé et irradié. 